\title{
On preventive blood pressure self-monitoring at home
}

\author{
Nervo Verdezoto $^{1} \cdot$ Erik Grönvall $^{2}$
}

Received: 1 March 2014/ Accepted: 8 November 2015

(C) Springer-Verlag London 2015

\begin{abstract}
Self-monitoring activities are increasingly becoming part of people's everyday lives. Some of these measurements are taken voluntarily rather than being referred by a physician and conducted because of either a preventive health interest or to better understand the body and its functions (the so-called Quantified Self). In this article, we explore socio-technical complexities that may occur when introducing preventive health-measurement technologies into older adults' daily routines and everyday lives. In particular, the original study investigated blood pressure (BP) measurement in non-clinical settings, to understand existing challenges, and uncover opportunities for self-monitoring technologies to support preventive healthcare activities among older adults. From our study, several important aspects emerged to consider when designing preventive self-monitoring technology, such as the complexity of guidelines for self-measuring, the importance of interpretation, understanding and health awareness, sharing self-monitoring information for prevention, various motivational factors, the role of the doctor in prevention, and the home as a distributed information
\end{abstract}

This work is based on an earlier work: "Understanding challenges and opportunities of preventive blood pressure self-monitoring at home", in Proceedings of the 31st European Conference on Cognitive Ergonomics (ECCE 2013), ISBN ISSN 978-1-4503-2251-5, (Aug 26-28 2013) (C) ACM, 2013. http://doi.acm.org/10.1145/2501907. 2501962.

Nervo Verdezoto

nervo@cs.au.dk

1 Department of Computer Science, Aarhus University, Aabogade 34 D, 8200 Aarhus N, Denmark

2 IT University of Copenhagen, Rued Langgaards Vej 7, 2300 Copenhagen S, Denmark space. An awareness of these aspects can help designers to develop better tools to support people's preventive selfmonitoring needs, compared to existing solutions. Supporting the active and informed individual can help improve people's self-care, awareness, and implementation of preventive care. Based on our study, we also reflect on the findings to illustrate how these aspects can both inform people engaged in Quantified Self activities and designers alike, and the tools and approaches that have sprung from the so-called Quantified Self movement.

Keywords Design Challenges $\cdot$ Home $\cdot$ Preventive care Self-monitoring $\cdot$ Home-based technology . Quantified Self

\section{Introduction}

Two modes of preventive self-monitoring have recently received attention from the media, business, and research: self-monitoring as part of a voluntary (in contrast to a referred treatment) and personal healthcare strategy, and self-monitoring as part of a personal interest in the socalled Quantified Self. Both modes of monitoring differ from tele-care (i.e. where measurements are sent to a healthcare professional, for example, at a hospital); as they are not prescribed, the measurements are not sent to a healthcare institution, and the activity is not done to monitor an illness, but to monitor health, well-being, and an individual's wish to learn about his or her body. A recent study in the United States of America (USA) confirms the trend, in which more and more people are starting to track health indicators for preventive reasons, and not only to manage chronic conditions (Fox and Duggan 2013). In particular, older US adults are more likely to track health 
indicators or symptoms (e.g. weight, diet, exercise, blood pressure, blood glucose) than younger adults. Furthermore, older adults are more likely to use pen and paper, and medical devices, to support their health-tracking activities. In contrast, younger adults keep their data mostly in their heads or on a mobile device (Fox and Duggan 2013).

To investigate older adults' preventive self-monitoring practices, our initial study was based on a project related to preventive blood pressure self-monitoring. High blood pressure (BP) is one example of an increasing age-related condition among older adults (WHO 2003). For a typical adult, high BP or hypertension is defined as a condition in which the systolic blood pressure (SBPblood pressure when the heart is beating) is equal to, or greater than, $140 \mathrm{mmHg}$ and/or a diastolic blood pressure (DBP-blood pressure when the heart is relaxed) is equal to, or greater than, $90 \mathrm{mmHg}$ (O'Brien et al. 2003). Measuring blood pressure outside of a clinic, for example, in a person's home, may be challenging, but also rewarding. Indeed, home-based self-monitoring technologies may be one way to support a continuously independent lifestyle among older adults, for example, by identifying potential risk factors before they become severe. Furthermore, home-based self-monitoring of diverse health parameters may also limit the "whitecoat" phenomenon (O'Brien et al. 2003). The "whitecoat" effect occurs when a person shows higher BP values at the hospital, owing to anxiety about being in a clinical environment, or vice versa (normal readings at the hospital and high values at home).

To help to understand the severity of high BP, we note that high BP is among the top five health risk factors in Denmark (Juel et al. 2008), and according to the World Health Organization (WHO), high BP increases the risk of heart disease and stroke (WHO 2003). WHO also defines high $\mathrm{BP}$ as one of the most prominent health risk factors in developed countries (among others, such as obesity, high cholesterol, and tobacco consumption) (WHO 2003). A Danish study revealed that $16 \%$ of men and $19 \%$ of women in Denmark have severe hypertension, and high BP has reduced the life expectancy of Danes by six to nine months (numbers from 2007) (Juel et al. 2008). To address challenges related to an increasingly elderly population, such as high BP, there is a neo-liberalist idealism that motivates Danish citizens to take responsibility for their own lives and healthcare management (Mik-Meyer and Villadsen 2007). Members of the Quantified Self community could, from one perspective, been seen to respond to this neo-liberalist idealism. However, people within the Quantified Self community monitor their bio-values not only because of an explicit desire to manage their health, but also to better understand and control their bodies and activities.
The Quantified Self community is typically composed of proactive people with a general interest in tracking all sorts of activities related to themselves, relying on the knowledge they can get from the measured parameters. In this community, it is common for its members to organize meetings to share and discuss their experiences and practices. Additionally, the members of this community have various types of motivators for tracking their values, such as finding new life experiences, improving several aspects of their lives, or improving their health status, in some cases (Choe et al. 2014).

Enabling individuals to be self-sufficient and sustain an independent lifestyle is one strategy for reducing healthcare costs (Coley et al. 1996). Shifting from disease to (proactive) individual health management promotes both home-based and preventive health care in society (Codagnone 2009; Tentori et al. 2012). Still, there are a number of measuring guidelines that must be followed to obtain correct measurements, and people may find it challenging to obey to these guidelines. In this article, we discuss challenges and opportunities related to the design of self-monitoring systems and services. We present aspects of preventive self-monitoring that emerged from our BP study, related to: the complexity of guidelines for self-measuring, the reliability of measurements, the importance of interpretation, understanding and health awareness, sharing self-monitoring information for prevention, various motivational factors, the role of the doctor in prevention, the use of technology for self-monitoring, the home as a distributed information space, and the visualization of health data. Then, these aspects are used to propose a set of design opportunities for both preventive healthcare self-monitoring and Quantified Self monitoring activities and technologies based on the results obtained from our study.

Before introducing our user studies, we discuss related research focusing on preventive care and preventive selfmonitoring, preventive self-monitoring technologies, Quantified Self and Personal Informatics, and challenges of self-monitoring.

\section{Related work}

This section describes research on the concept of preventive care, Quantified Self research, and introduces both preventive and Quantified Self monitoring tools and technologies.

\subsection{Preventive care}

Mallery and Rockwood (1992) define prevention (in a healthcare context) as any action that "delays disease or 
slows its progression" (Mallery and Rockwood 1992, p. 2371). Preventive health measures include primary, secondary, and tertiary interventions (Stults 1984). Primary prevention focuses on decreasing the risk of an illness appearing (i.e. immunizations and health promotion). Secondary prevention focuses on early detection and treatment, especially for asymptomatic diseases (e.g. cancer, hypertension, and dementia). Tertiary prevention focuses on rehabilitation to slow deterioration and decrease disability caused by symptomatic but unreported illnesses, by using a comprehensive assessment of functions (i.e. physical, psychological, and social) (Kane et al. 1985; Mallery and Rockwood 1992; Stults 1984).

Kane et al. (1985) present four main areas of intervention for preventive strategies: (1) specific conditions or illnesses that result in dysfunction (e.g. cardiovascular disease, cancer), (2) risk factors that may be categorized as unhealthy behaviour, rather than illness (e.g. smoking, lack of exercise, unhealthy diet), (3) conditions that require caregivers' intervention (e.g. depression, drug abuse, visual and hearing impairment), and (4) dealing with consequences of caregiving and medical care (e.g. drug side effects, functional disability in overprotective environments) (Kane et al. 1985). Furthermore, it is important to understand the differences and similarities between prevention and promotion in health care (Czeresnia 1999). Many health promotion measures (e.g. for older adults) are related to primary or tertiary prevention and aim for behavioural change in older adults at risk, or to improve their functional capacity (Czeresnia 1999; Mallery and Rockwood 1992). However, lifestyle changes or preventive care activities (e.g. healthy diet, regular exercise, avoiding alcohol, or smoking) may be sufficient for some, but not all, healthy older adults. As older adults are not a homogeneous group, preventive care that aims to modify a risk factor after its effects have been identified may not be effective, especially for frail older adults (Kane et al. 1985). An older adult with a diagnosed symptom may need monitoring and individual assessment, including medication adherence support, to control a specific condition [e.g. high BP (WHO 2003) or diastolic dysfunction (Epstein and Wei 1992)], and avoid excessive or ineffective treatment (O'Brien et al. 2003). For example, people over 80 years old are more likely to suffer from diastolic issues (a lack of arterial-blood oxygen when the heart is relaxed) (Epstein and Wei 1992).

Similarities and differences between chronic illness management and prevention do exist and have been analysed by Glasgow et al. (2001). They also investigated whether or not the chronic care model (CCM) proposed by Wagner (1998) is suitable for prevention. The similarities include: (a) ongoing planned care with "proactive followup"; (b) complexity, owing to multiple health behaviour changes or risk factors; (c) illness/reactive care is inadequate; (d) active patient involvement is required; and (e) patient activation to recognize and take individual action towards care activities (Glasgow et al. 2001). The differences include: (a) visits to the doctor for prevention are less frequent than for chronic illnesses; (b) patients with chronic illnesses have stronger motivation to change health-related behaviour; (c) patients' care needs are greater for chronic illness care [e.g. medication adherence (Dalgaard et al. 2013a)]; (d) prevention often happens outside the medical culture; and (e) prevention is less often tracked (Glasgow et al. 2001). Although differences and similarities do exist, Glasgow et al. (2001) suggest that an extended version of CCM may be suitable for understanding, improving, and developing preventive care. Our study recognized these similarities and differences to better understand preventive self-monitoring practices at home.

\subsection{Preventive self-monitoring}

When compared to disease management, preventive health care has been less researched (Codagnone 2009). However, examples do exist, such as Beaudin et al. (2006), who use mock-ups of diverse data visualizations to reveal user reactions to home monitoring for proactive health care and well-being. One of the most common examples of prevention is accident detection (e.g. falls), which has been addressed using sensors and algorithms (Fahmi et al. 2012). Self-monitoring of health parameters as part of primary prevention has been tightly coupled with health promotion and challenges related to lifestyle and behaviour changes (Klasnja et al. 2011). In secondary prevention, the concept of embedded assessment has been introduced by Morris et al. (2005), to promote the early detection of declining health. Early detection should integrate monitoring, compensation, and prevention, to provide value beyond the assessment, to address clinical barriers (e.g. infrequent, delayed, and limited focus of assessment) and individual barriers (e.g. privacy concerns, complex terminology, selfmonitoring device constraints, fear of diagnosis of diseases) (Morris et al. 2005). Additionally, tertiary prevention is exemplified by monitoring how often and how well instrumental activities of daily living are performed by ageing adults (Lee and Dey 2010).

\subsection{Preventive self-monitoring technology, Quantified Self, and Personal informatics}

We now sequentially present the three related topics of preventive self-monitoring technology, followed by a section on Quantified Self, and one on Personal informatics.

Technology to support preventive self-monitoring strategies, as part of a voluntary and personal healthcare 
interest, often makes use of technology that is on the market. Traditionally, devices to support preventive strategies are designed and developed for the medical domain, and they have already been tested and are accessible as off-the-shelf products. Examples of such products include blood pressure monitors, glucometers, ECG, INR metre, peak flow meters, and pulse oximeters. One special characteristic of the medical devices is their strong focus on the reliability of the measurements, and they have been put on the market after appropriate testing. In addition, some commonly used devices, such as the body weight scale, are developed for personal care, rather than for medical reasons (Ashiful et al. 2012; Baker and Kirschenbaum 1993; El Assaad et al. 2003).

Although much research and product development take place within the clinical field, there is also the parallel development of a less formalized, non-institutional, "grassroots" movement interested in quantifying the body (Smarr 2012). Quantified Self enables "self-knowledge through numbers" (Rivera-Pelayo et al. 2012, p. 1). Quantified Self is a movement, a set of tools, and a community interested in better understanding the body and its physical and mental functions and activities through biomeasurements. Although self-tracking tools often focus on recording bodily functions, Quantified Self has a broader and voluntary focus that aims to record and quantify almost every aspect of everyday life with the goal of self-improvement (Lupton 2013b, c). Ubiquitous computing (UbiComp) technologies and smartphone apps provide new ways for people to track their behaviour more frequently than before, fostering personal reflection and self-discovery. Although it cannot be expected that self-dedicated technology and practices will eliminate illness, it may help to reduce global healthcare costs through the resulting preventive reflection and actions (Hood and Flores 2012; Smarr 2012).

Through the development of mobile phones, with more and more sensors embedded in each new model, more data may be obtained "by everyone" (Smarr 2012). More and more special-purpose and commercial body and health parameter measuring tools also exist. Personal Informatics tools are the ones that enable people to collect personal information and facilitate their reflection on their behavioural data ( $\mathrm{Li}$ et al. 2010). Rather than being just a class of personal tools, Li et al. (2011) note that these further extend the concept of Personal Informatics to an "activity", with the same aim of supporting the collection of, and reflection on people's personal data. Self-tracking and Quantified Self technologies (e.g. Fitbit, Jawbone UP, NikeFuel, WakeMate, Withings Scale) belong to these types of systems that focus on data collection and quantification of a variety of everyday activities (e.g. work, leisure, and self-care) such as diet, exercise, sleeping patterns, weight, blood pressure, hearing function, work productivity, social behaviour, and alcohol and tobacco consumption (Lupton 2013b; Swan 2012; Wiederhold 2012; Yau and Schneider 2009). The Quantified Self movement is rather experimental by nature, and the movement's followers measure, test, and reflect on their bio-values over time (Rivera-Pelayo et al. 2012). Hence, Quantified Self is empirical, rather than built on proven research models (Rivera-Pelayo et al. 2012).

\subsection{Challenges of self-monitoring}

While typical medical devices for self-monitoring have been designed according to the medical model driven by health professionals (Storni 2013), Personal Informatics systems and Quantified Self tools have a strong technological focus on system design (Swan 2012) and often neglect the medical perspective. Although these technologies have facilitated and automated the data collection for self-monitoring, both medical devices and Quantified Self tools often neglect the individual or "citizen perspective" (Ballegaard et al. 2008, p. 1809; Storni 2013). In particular, home-based monitoring technologies have not recognized the complexity of the lived experience (Fitzpatrick 2011; Lupton 2013a; Verdezoto et al. 2014) while monitoring health and disease, which goes beyond self-monitoring, in many ways (Grönvall and Verdezoto 2013). When using self-tracking technology for self-care, both the individual and the medical perspectives should be considered in system design (Das and Svanæs 2010). Following this initiative, for example, Quantified Self tools present problems regarding the accuracy of the self-measured values. For instance, Montgomery-Downs et al. (2012) report a study on the validity and reliability of Fitbit, regarding activity monitoring in which they report an overestimation of sleep time and quality, owing to a misidentification of the waking pattern as sleep. Intensive validation is needed, if devices such as Fitbit are to be used for self-care, especially by populations with specific and critical health needs (Montgomery-Downs et al. 2012). Furthermore, the strong emphasis on quantification of health outcomes, such as achieving dietary or glucose-level goals, may hinder the real value of self-monitoring. For instance, TurnerMcGrievy et al. (2013) report no difference in health outcomes of diet and physical activity, when using automatic or manual self-tracking tools. As the numeric values are one of the aspects that support self-learning and self-reflection (Grönvall and Verdezoto 2013), most of these technologies have neglected the inherent qualitative aspects of self-reflection, whereby the numeric values are interpreted and given meaning (Lupton 2013c; Swan 2013). 
The foregoing technologies may not be enough to support preventive health self-monitoring activities, as this may require: (1) a better understanding of preventive selfmonitoring needs while investigating the lived experience of the individual (Ballegaard et al. 2008; Fitzpatrick 2011; Storni 2013) and (2) a proactive activation of people's selfcare abilities (Glasgow et al. 2001; Wagner 1998) for prevention, to complement the role of the "informed, activated patient" (Wagner 1998), when considering multiple perspectives on self-care (Das and Svanæs 2010). A lack of consideration of multiple perspectives on self-care and an overemphasis on quantification may cause unintended side effects, such as obsession and stress, rather than empower people with self-care (Lupton 2012, 2013a; Storni 2010). A negative health outcome may be the result, as shown by a recent report, in which healthy, overweight people who lost weight had a $15 \%$ higher mortality rate than those who maintained their weight (Sørensen et al. 2013).

\section{Method}

This study integrated qualitative studies and a quantitative survey in three main steps. First, an initial and exploratory phase defined a relevant target group, using interviews and cultural probes. Second, we selected a specific health parameter of interest for our study (i.e. blood pressure), and we conducted several workshops, semi-structured interviews, and blood pressure self-measurement activities in people's homes. The third and final step was a quantitative study to confirm and further explore preventive self-monitoring activities in the Danish population. These three main steps are described in the following subsections.

\subsection{Defining the target group}

An initial qualitative study including ten participants (four women and six men) with an average age of 61.8 years (SD 11.95) took place during the project's start-up phase. Since chronological age was not a central question, participants were selected according to their relationship to the Danish healthcare system, matching the three levels of preventive health measures [i.e. primary, secondary, and tertiary prevention (Stults 1984)]. Four participants were categorized in the primary prevention group (one healthy without a diagnosis and three taking preventive medication and at risk of going into treatment), three participants were categorized as belonging to the secondary prevention group (people with chronic diseases, but that have changed their lifestyles to prevent further disease-related deterioration), and three participants in the tertiary prevention group (two in physical therapy after arterial thrombosis and one former cancer patient). Participants were interviewed about their personal motivation regarding health, in relation to their knowledge of their current health status, their health data sharing needs, what motivated them to change behaviour, their use of self-monitoring technologies at home, and their experience with self-monitoring technology, perceived as beneficial or not. Additional material (photographs taken by the participants using disposable cameras) was collected as probes.

\subsection{Exploring challenges through a case study of preventive blood pressure health monitoring}

Based on findings from the previous step, the project group decided to focus on people that are motivated to maintain an existing healthy status, and avoid deterioration or the onset of illness, that is, prevent frailty. The project then recruited a new group of participants from a local senior activity centre. The participants were ten active older adults that perceived themselves as relatively healthy. Participants were between 65 and 84 years old. At the activity centre, our work extended into a user-involved design process, including interviews and workshops with the older adults. Together with the older adults, the project team investigated challenges and attitudes towards the preventive action of blood pressure self-monitoring. Blood pressure was selected for analysis of self-monitoring, owing to its complexity (number of steps to follow in order to record a valid measurement), importance (relationship to other risk factors), and device availability (e.g. presence of blood pressure measuring devices at older adults' homes), identified through the initial photographic probes. Additionally, a nurse was involved in the project and participated in the first workshop. Two of the participants left the project after the first workshop, owing to the detection of high BP. All sessions were recorded and took place at the senior activity centre.

\subsection{Quantitative study for further exploration and confirmation of preventive self-monitoring activities}

To include a broader population to further explore selfmonitoring activities and the workshop results, the project partners conducted a quantitative study. A web survey was developed and distributed through the formal project partner's social media networks.

The questionnaire included 29 questions that helped to better understand the following eight areas: (1) overall health status, (2) technology and device ownership, (3) sharing of generated data, (4) perceived gains from using self-monitoring technology, (5) use of technology, (6) practical use (of technology), (7) home security-related 
technology (burglar alarms, etc.), and (8) level of physical exercise. The survey also included questions regarding personal information (e.g. age, gender, location). We received 191 responses to the survey, but only 165 were completed and hence considered valid for our analysis. The survey participants were adults between 22 and 83 years old and were from all regions of Denmark. The capital and two (of five) regions had significantly higher numbers of returned surveys.

\subsection{Data analysis}

Our empirical material from our studies was analysed using both inductive and deductive content analysis. A deductive approach is a top-down, analytic-driven way to provide a more focused analysis of specific aspects of the data, whereas an inductive analysis is a bottom-up approach that relies on the themes that are identified in the data (Braun and Clarke 2006). In our study, the most relevant themes come from our diverse studies and workshop activities (inductive), on the one hand, and from the accounts of the most relevant challenges of the related work (deductive) that is presented in Sect. 2.4 (e.g. lack of understanding, privacy, aesthetics issues with self-monitoring devices, difficulty in understanding clinical terminology, people's attitudes and perceptions about self-monitoring, problems with measuring devices) (Ashiful et al. 2012; Morris et al. 2005; Parati et al. 2010; Tentori et al. 2012). Additional interviews were conducted with two participants, to confirm some results of the analysis. The themes were iteratively identified by the two authors and discussed with the other members of the project, especially the project leader and the ethnographer involved. Themes were consistent among the participants in the various studies, and the diverse themes highlight particular aspects of self-monitoring that challenge self-care activities in non-clinical settings.

\section{Case study: preventive blood pressure health monitoring}

After defining the target group as perceived healthy older adults, we started exploring an initial set of possible health parameters to further investigate the challenges of selfmonitoring at home. We initially reviewed a list of health parameters suitable for self-monitoring, provided by one of the project partners (Verdezoto et al. 2012). These health parameters are summarized in Table 1 and included parameters such as peak expiratory flow, systolic and diastolic blood pressure, and blood glucose level. These parameters are arranged in various categories that refer to specific medical conditions for which these health
Table 1 Sample of health data

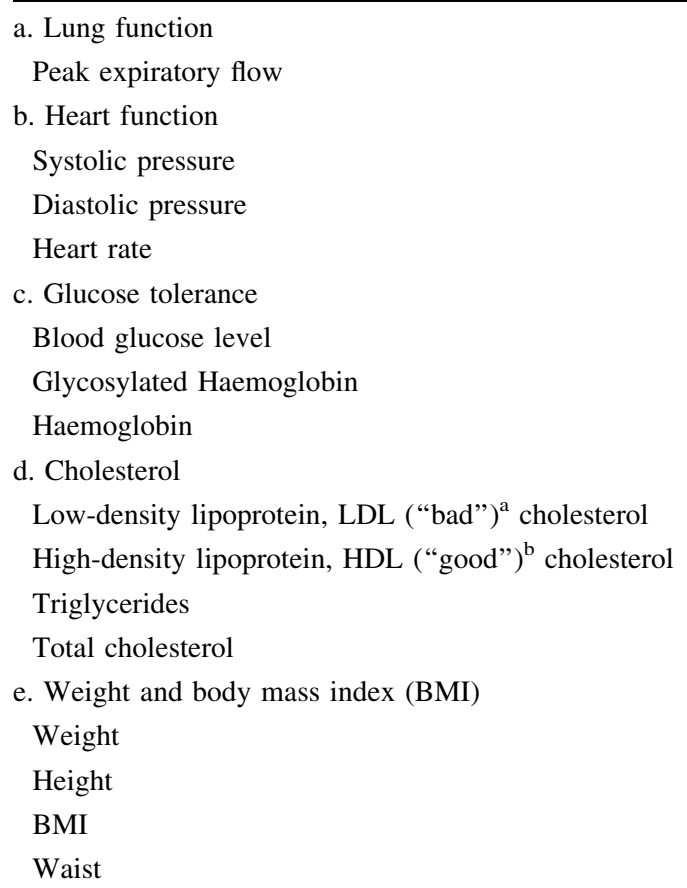

parameters are usually collected and monitored, such as (a) asthma and COPD, (b) hypotension, prehypertension, hypertension, and hypertensive crisis, (c) hypoglycaemia and hyperglycaemia, (d) hypercholesterolaemia, and (e) obesity. From this initial set of health parameters, we purposefully selected blood pressure (BP) for further exploration, owing to: (1) the level of complexity involved in taking the measurement, based on the number of steps a person must follow to obtain a valid measurement, (2) the relevance of $\mathrm{BP}$ as one of the most important risk factors in Denmark, and (3) the presence of BP measuring devices in older adults' homes during the initial qualitative study.

After selecting BP for further exploration, we conducted several workshops to investigate the challenges and opportunities to support preventive self-monitoring activities. Workshop activities explore why, how, and when preventive self-monitoring activities could be performed as part of everyday activities, and are presented in detail in the following subsections.

\subsection{Workshop \#1: understanding self-monitoring activities}

An early goal of the project team was to get a better understanding of self-monitoring activities, and their importance with regard to older adults' health. The workshop participants received an introduction to BP (causes, 


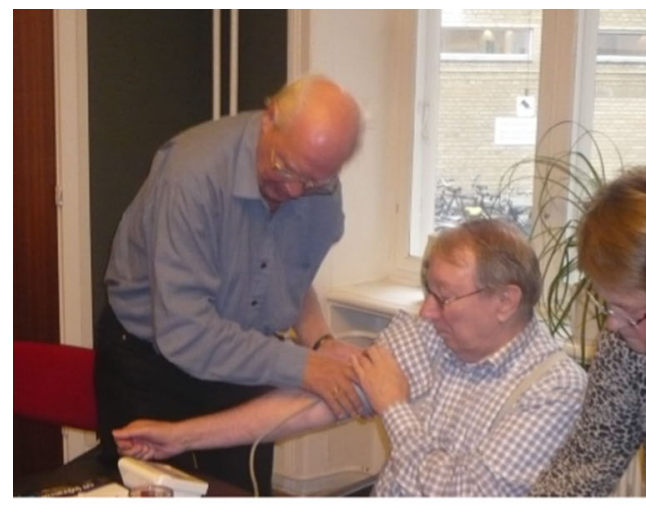

(a)

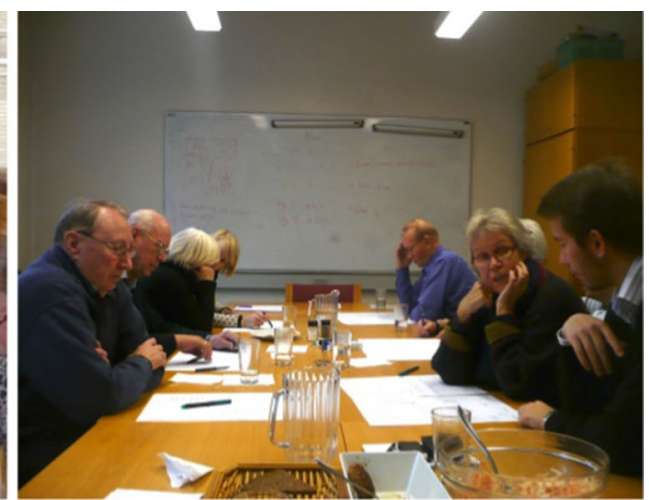

(b)

Fig. 1 Workshops: a understanding self-monitoring activities; b understanding older adults' routines

symptoms, consequences, and actions to control BP) and to measuring BP, by the nurse affiliated with the project. During the workshop, participants were asked to take their own BP (see Fig. 1a), and they all engaged in the activity, using a digital BP measuring device. A group discussion was held during and after each activity. Findings from the first workshop indicated that the complexity of the BP measuring guidelines challenged self-monitoring activities.

\subsection{Workshop \#2: understanding older adults' routines}

A second workshop was conducted two weeks after the first. We employed a modified version of the day reconstruction method (DRM) (Kahneman et al. 2004), both asking participants to recall activities, and link activities with places in their homes, to further investigate older adults' daily routines. We applied the modified DRM to explore difficulties and possibilities of integrating BP measurement activities into everyday routines.

As part of the workshop, participants engaged in a drawing and reflection activity about their homes and daily routines (see Fig. 1b). The workshop participants were asked to: (a) sketch floor plans of their homes (see example in Fig. 2a), (b) list their activities before they leave home (in the morning, or when they consider their morning to be concluded) and after they return home (in the afternoon, or when they perceive that they started their evening activities), and (c) order the activities using numbers on the drawing. One of the researchers gave an example of how to sketch the floor plan and how to list the activities before the drawing activity started. One participant needed assistance from a researcher to perform the task. After the initial sketching activity, the participants verbally presented and shared their sketches and routines. Once all participants shared their sketches and routines, a group discussion followed, about the possibilities and implications of using

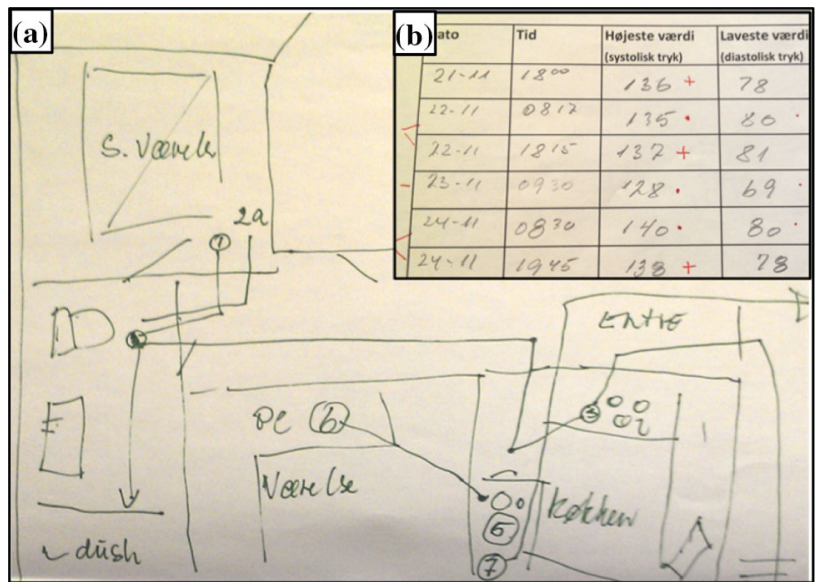

Fig. 2 a Floor plan sketch of a participant's home; b a paper-based template for registering the BP measurements

self-monitoring technologies throughout the day, and how measurements could affect daily activities.

Findings from the second workshop revealed differences between older adults' morning and evening routines. The evening routines were generally more diverse among the participants and less stable, owing to the older adults' active lifestyles. Therefore, a further investigation into how older adults might perform BP self-monitoring at home was initiated. The participants were requested to take BP self-measurements at home until the next workshop, \#3 (Sect. 4.3). The participants were provided with digital BP measuring devices and were asked to measure and record their BP for at least three days in a row (mornings and evenings), during a one-week period (as suggested by the nurse, according to existing guidelines on how to take a correct BP measurement at home) (Parati et al. 2010). The participants were given these guidelines (e.g. do not smoke, drink coffee, eat, or consume alcohol in the half-hour before measurement) verbally and in printed form. The participants also received a printed template on which they 
could register their pulse and $\mathrm{BP}$ values, together with the date and time of the measurement (see Fig. 2b). BP measurements are registered as systolic blood pressure (higher value) and diastolic blood pressure (lower value). Furthermore, the template included a field for additional comments (e.g. which arm they used).

\subsection{Workshop \#3: validation and interpretation of BP values}

To share their experiences of self-monitoring and to validate previous findings, the workshop participants were invited to a third workshop. At this workshop, the participants presented the collected BP information, the BP measurement guidelines they had applied, and the difficulties they experienced when performing the measurement task.

Next, we wanted to investigate what and how BP information may be presented to older adults in an understandable way. The participants were given eight different colour image pairs, combining weekly and daily measurement overviews, to elicit feedback regarding visualizing data from self-monitoring technologies, as suggested by Beaudin et al. (2006). Examples of these weekly and daily overviews are illustrated in Fig. 3. For example, the weekly BP overviews were represented in three different formats, using: (1) colour-coded charts with icons, circles, starts, or squares, not specifying exact values (e.g. see Fig. 3a); (2) vertical and horizontal bars (see Fig. 3b); and (3) line charts (see Fig. 3c). The daily overviews were represented by four different formats, using: (1) icons to represent specific BP values (see Fig. 3d); (2) text values of
BP (see Fig. 3e); (3) speedometers visualizing BP values (see Fig. 3f); and (4) colour-coded bars representing BP values (see Fig. $3 \mathrm{~g}$ ). These weekly and daily visualizations were combined, one pair was shown at a time, and the participants commented and gave feedback on the presented visualizations. To conclude the workshop, the participants engaged in a group discussion about how to visualize and facilitate the interpretation of health data for individuals that have no previous knowledge of medical data.

\section{Results: aspects of preventive self-monitoring}

In our quantitative survey, 131 respondents (of 165) had no chronic disease (see Fig. 4a), and 86 of these participants owned a health self-monitoring device (see Fig. 4b). This suggests that people without chronic conditions are interested in their health and desire "to stay healthy" as long as possible.

In detail, the quantitative results show that $17 \%$ of the men and $28 \%$ of the women in the sample population had one or more chronic conditions. The most common conditions were COPD, diabetes, asthma, allergies, and heartrelated problems. Between the people with and without a chronic condition, the most common self-monitoring technologies were heart rate monitors (11 of those with a chronic condition and 48 of those without) and pedometers (12 of those with a chronic condition and 38 of those without). Many also had scales with integrated BMI measuring (eight of those with a chronic condition and 22 of
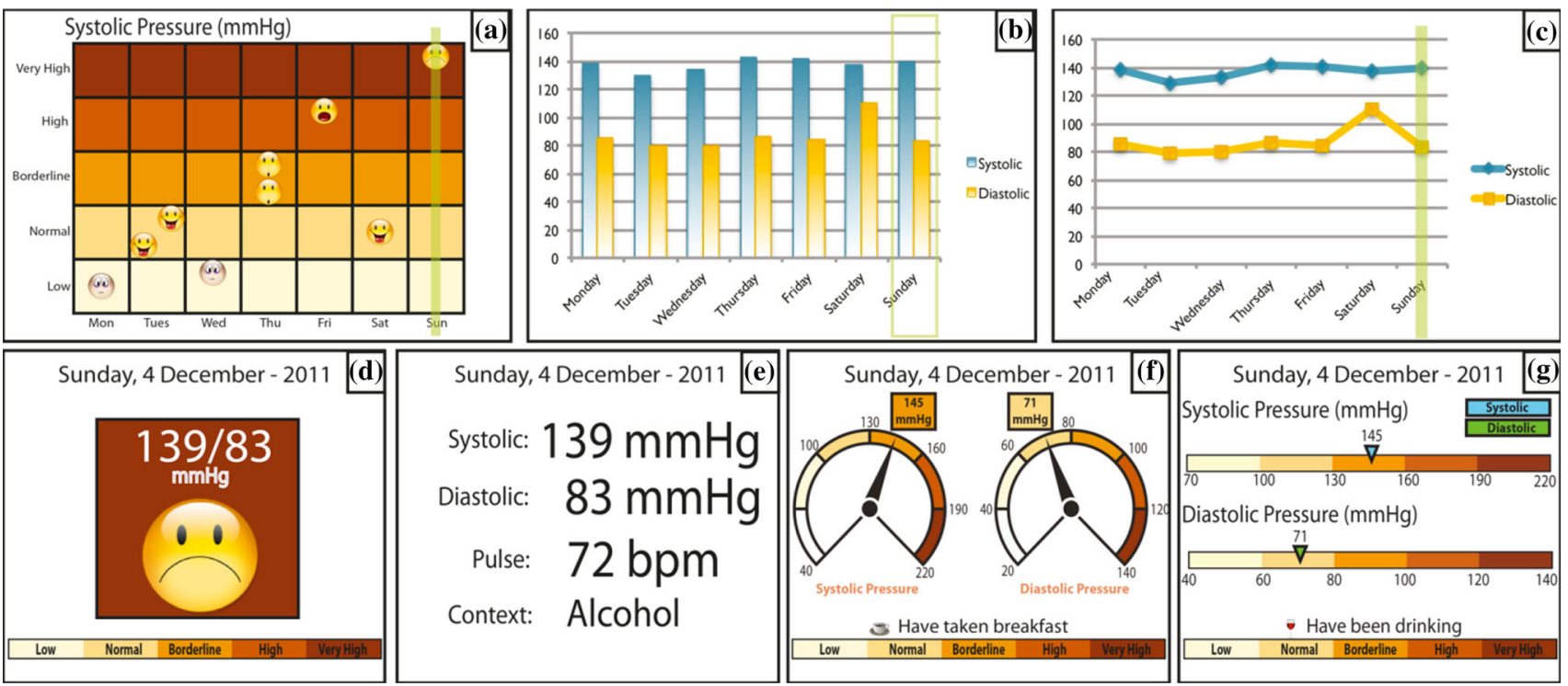

Fig. 3 Examples of visualizations using: a icons for overview; b bar graphs for overview; $\mathbf{c}$ line charts for overview; d icons for details; e text for details; $\mathbf{f}$ speedometer for details; and $\mathbf{g}$ colour-coded bars 
Fig. 4 a Number of participants with and without a chronic condition; $\mathbf{b}$ number of participants who own a health self-monitoring device

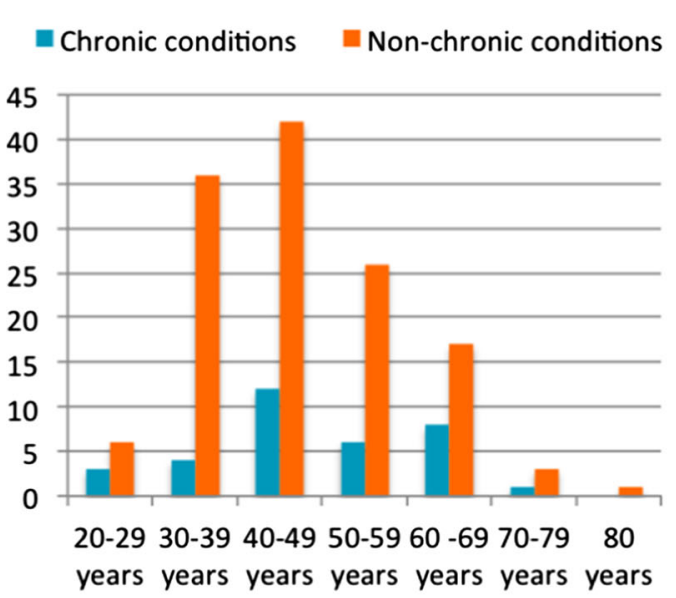

(a)

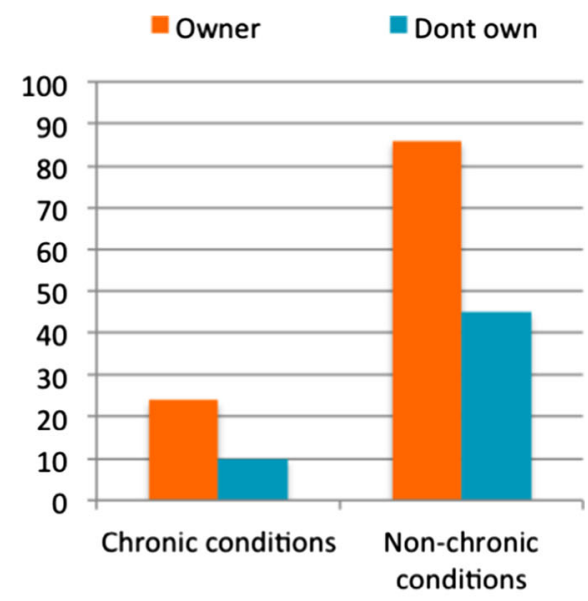

(b) those without) or BP measuring devices (ten of those with a chronic condition and 26 of those without).

The survey results do not reveal a clear difference between the chronic and non-chronic groups, with respect to whether they own at least one health self-monitoring device at home. Among the 110 responders who did own a self-monitoring device, 43 said they used it for prevention purposes and 42 said they used it because of a general interest in health. Eleven participants replied that they monitored because of the need to track a chronic condition. The others monitored for "other" reasons. Among the responders, $77 \%$ could see themselves using self-monitoring technology to supplement existing health services and $60 \%$ were open to using self-monitoring instead of existing services. The data do not indicate a correlation between the willingness (or lack thereof) to use self-monitoring technology instead of existing health services, and the responder's age. However, there was a strong tendency for participants to dislike sharing their data, or to share it with their general practitioner only. Also, $16 \%$ would like to share their data with homecare workers, $33 \%$ with healthcare professionals (other than their GP), and $14 \%$ would be open to the idea of sharing these data with their family and friends.

Nine themes were identified during the analysis, highlighting particular aspects of preventive self-monitoring that are important to consider when designing self-monitoring technologies that would integrate into people's everyday lives. The nine themes are: (1) the use of selfmonitoring technology, (2) the complexity of measuring guidelines, (3) reliability of the measurements, (4) interpretation, understanding, and health awareness, (5) visualization of BP values, (6) the home as a distributed information space, (7) sharing self-monitoring information for prevention, (8) motivational sources, and (9) the role of the doctor in prevention. We now present the nine challenges that emerged from our study.

\subsection{The use of self-monitoring technology}

The workshop participants had a rather general understanding of technology. They all had mobile phones and modern household appliances. They were interested in computers to some extent, as they all took a beginner's computer class at the senior centre where we arranged the workshops. The participants (except for one, who later withdrew from the project) did not have previous experience using a BP measurement device at home. They all could operate the digital, off-the-shelf BP measurement device after its introduction in the first workshop. However, there is a difference in being technically able to operate a device and to actually obtain valid measurements (e.g. to comply with all guidelines related to a valid BP measurement). Although usability is a main concern in most system designs, especially for end-user or consumer products, the study reported here did not directly investigate usability and interface issues. Instead, the main interests of this study were on challenges, and user experiences when taking correct BP measurements, and how to support correct data generation. Our study addressed possible concerns that may influence the quality of the measurements, once the device itself is correctly operated. During the first workshop, all participants learned the guidelines that exist to guarantee a valid BP measurement.

From the quantitative studies, we could see that healthmonitoring technologies have a presence in people's homes. Heart rate monitors, BP monitors, and pedometers were the most frequently available devices among the 131 respondents without chronic conditions. Furthermore, they preferred that measured data were recorded automatically. Data that the participants suggested to be recorded were (number of respondents, within parenthesis): (1) physiological parameters, such as weight and blood pressure (89), (2) diet (74), (3) well-being parameters, such as depression and stress (44), and (4) cognitive functions, such as 
memory and analytical skills (43). Twenty-four participants replied that they would not like to measure any health data about themselves. Additionally, BP measuring devices were found in people's homes during the initial qualitative study, through the use of cultural probes. This confirmed our selection of BP as the main health parameter for this study.

\subsection{The complexity of measuring guidelines}

An important aspect of preventive BP self-monitoring is the complexity of the measuring guidelines. This complexity relates to the guidelines or recommendations to which someone must adhere, to guarantee a valid (BP) measurement. For example, one should sit and rest for 5 min before measuring BP. Our workshop participants mentioned several difficulties in measuring BP in accordance with the established guidelines. A common issue discussed among workshop participants was that they did not (in their perception) have enough time to correctly take a measurement (and hence, apply to the guidelines of resting before taking a $\mathrm{BP}$ measurement, or other timeconstraining guidelines, such as not eating, drinking, or smoking $30 \mathrm{~min}$ before the measurement). Indeed, older adults have other activities than self-monitoring to attend to during the day.

When examining the self-collected data from the monitoring activity at home, it was clear that all workshop participants took their morning BP measurements. However, three workshop participants experienced difficulty in integrating the evening measurements into their routines and hence omitted one or more of these measurements. For these participants, developing and adjusting routines to suit the BP measurement guidelines were complicated during the evening, owing to active lifestyles, for example. Indeed, a male participant with a very active social life could not find the time to carry out the preventive measurement task for three days (two measurements a day) in a row (this configuration was suggested by the project nurse). He said "I cannot do it this week [looking at his agenda], maybe next week I can try..." Analysing the workshop participants' floor plans of their homes and the activities situated therein confirmed the characteristics of morning and evening routines. In general, we found that the morning routines were better established than the evening routines, as they appeared more stable, and were easy for all participants to recall.

\subsection{Reliability of the measurements}

The reliability of measurements taken by laypeople in their homes is here in foci. Owing to the complexity of the guidelines when conducting valid home-based measurements, and the lack of contextual information (e.g. whether a person rested, talked, consumed alcohol, or smoked before or while taking the measurement) that might indicate failure to follow the guidelines, for example, the reliability of the measurements may be compromised. For instance, the participants had to take more than one BP measurement to ensure and verify their measurements. Four workshop participants took the measurements on one or both arms (this being contextual information), two or three times in a row, during the home self-monitoring task to assure and verify their measurements. Even though we asked participants to write down all contextual information, the only information that a few of them wrote down was about the arm from which the measurement was taken, because they stated that most of them did not remember to write down such situations. In addition, one participant wrote down a technical issue with the BP self-monitoring device related to low battery. When the contextual information is missing or misleading, readings may show unexplained erroneous values that might not accurately reflect the current situation. For example, the readings may show stage one hypertension, but this could be related to missing contextual information (e.g. the participant did not follow the guidelines and did not report non-adherence to the guidelines) related to the measurement. We also observed that measurements could be accidentally taken incorrectly, for example, owing to an incorrectly placed cuff. Additionally, we found possible misreporting owing to handwriting mistakes (done by two participants) or a malfunctioning device (the battery issue reported by one participant).

\subsection{Interpretation, understanding, and health awareness}

People must not only become aware of and understand the consequences of unintentional mistakes or misreporting that may compromise the reliability of measurements, but also they must be able to interpret and understand the meaning and implications of the measurements. During the initial qualitative study, a participant said, "the scale shows too much. Could my weight gain be a side effect to the pills? I can feel and see that I have gained weight". From our workshop activities, it became clear that all participants were well aware of, and understood, the importance of lowering their BP if they had elevated BP. However, methods for lowering BP were less understood, and the workshop participants were interested in learning more about how they could decrease their BP values. In general, all workshop participants could use the BP measuring device we provided, after limited initial training. However, moving from correct device usage to a proper understanding of the measurements (i.e. the numeric values of 
the BP) was more challenging. Although the participants managed to take proper measurements, some of them failed to understand or interpret the measured values. For instance, half of the participants had a BP value over the normal level when they made their first measurement during the first workshop, and therefore, the nurse asked them to repeat the measurement on the opposite arm, to compare the BP values. The nurse suggested that the lower value (taken from either of the two arms) should be considered the reference point for future measurements. The nurse stated that a higher value from a specific arm is due to an obstruction of the arteries in the side of that arm. The nurse played an important role, explaining the meaning of the values from each arm and the importance of taking the measurements at least twice a day (morning and evening), three days in a row.

When reporting the BP measurements using the printed templates provided, one workshop participant used symbols (e.g. lines and "+" signs) to group them and identify values within the normal BP range. In addition, three workshop participants took additional readings from both arms, to compare and understand the BP values, thereby supporting the interpretation. Furthermore, an understanding of their current health status may support older adults in achieving a sense of health awareness, in terms of maintaining or achieving an improved health status. A participant from our qualitative study stated, "For many years I forgot about exercise. 5-6 years ago I had a slipped discs. I have avoided surgery with the help of painkillers and exercise". In general, we confirmed that high blood pressure is indeed a non-communicable disease, as people may not be aware that they have high BP. The non-communicable aspects of high BP became clear in our study, as two participants left the project after they became aware that they had high BP after the first workshop.

\subsection{Visualization of BP values}

Our workshops revealed that older adults have some difficulties in understanding BP readings. Even though they could take the measurements, they experienced difficulties in interpreting the resulting values (as described above). Thus, we explored various ways to enhance their understanding of BP data, using visualizations. In this activity, we explored two levels of information, the weekly overview and the detailed view of measured values from the current day, as illustrated in Fig. 3.

\subsubsection{Weekly overview}

First, workshop participants found the visual coding of the charts useful. They used it as an indicator of the meaning of the various BP values, such as "low", "normal", "borderline", "high", and "very high" (see Fig. 3a). A workshop participant stated, "It is simple and easy to understand..." However, they felt that the charts were not precise when the BP values were not shown, and they liked the combination of measured values and line charts (see Fig. 3c). Second, participants found the simplicity of the line chart useful. However, one workshop participant did not find the dots in the curve to be relevant. For example, one participant commented, "... maybe you could add the smiley faces to the curve". This presupposes that people might need to know the various meanings of the smiley faces, if they were associated with the home-based measurements. Third, workshop participants agreed on a simple way to see the actual value of the measurement through the bar graphs (see Fig. 3b). However, two workshop participants did not understand the meanings of the values and suggested that the bars could have additional indicators to represent the meaning of the value.

\subsubsection{Daily details}

First, workshop participants found the icons useful, but also indicated some problems with the iconic data representation (see Fig. 3d). A workshop participant stated, "It is simple, appealing and funny, and it is easy to understand, but not precise". Second, workshop participants relied on text values, as this allowed them to simply extract and see the actual measurement value (see Fig. 3e). Third, one workshop participant found the speedometer useful (see Fig. 3f), saying that it is "kind of" relevant since blood pressure is like a speedometer. This participant commented, "It [the blood pressure] goes up and down". However, workshop participants also agreed that in some cases, a speedometer might not be precise. Finally, workshop participants found the colour indicators of the colourcoded bars- "red-toned bars"-useful, and perceived them as a good strategy for showing the daily measurements. A participant said, "The idea of the daily overview is kind of ok" (see Fig. 3g).

\subsection{The home as a distributed information space}

From our initial qualitative study, we observed how people and everyday objects are immersed in various spaces in which the information may be distributed and interchanged in many ways, according to people's routines, activities, and preferences. In a distributed information space, people and artefacts may be either mobile or fixed agents, across various operation centres, where activities take place (González et al. 2005). From our data, we identified several operation centres at home that are dedicated spaces for specific activities, such as the kitchen, bathroom, dining room, living room, garage, and bedroom. 


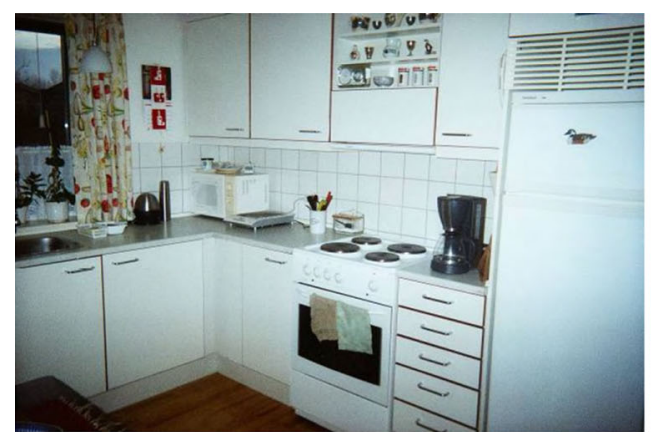

(a)

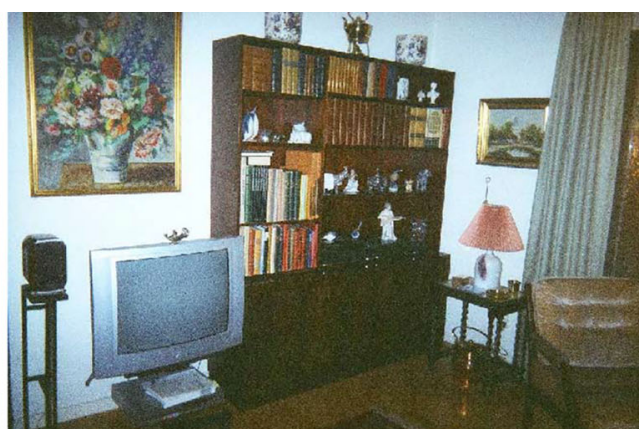

(b)

Fig. 5 Examples of operation centres at home: $\mathbf{a}$ the kitchen and $\mathbf{b}$ the living room

Specific activities are performed in these operation centres, such as cooking in the kitchen and watching TV in the living room (see Fig. 5). For instance, one of the participants from the qualitative study stated that she has the TV on almost all the time, as it gives her a feeling of safety while performing everyday activities. Indeed, people can perform other activities in these spaces, or various everyday objects may be moved about the home, bringing a different meaning with them. For instance, when the workshop participants drew sketches of their homes, it was possible to observe how participants visualized and communicated their routines across various spaces in their homes. Analysis of the drawings disclosed four similarities of participant behaviour: (1) there are common places for specific routines among the participants (e.g. brushing their teeth or hair happens in the bathroom), (2) morning routines are more established than evening routines (as explained above), (3) activities may occur in one or more places (e.g. listening to the TV while reading the newspaper in another room), and (4) more than one activity may take place at the same time (e.g. eating breakfast and reading the newspaper).

When an object is moved into a different space, it may change its meaning, as it migrates into a different context. One particular example of an object changing meaning as it is moved into different contexts is the distribution of medication across various spaces in the home, according to people's routines (Dalgaard et al. 2013a). Figure 6a illustrates a situation in which some medications were on top of a washing machine in a bathroom, whereas other medications may be on a kitchen cabinet, or in a bedside table, according to the routines. Another example is illustrated in Fig. 6b. Here, the small heart-shaped box has sentimental meaning, and it has been brought to the kitchen to provide a feeling of safety, as it has a specific emotional value for the participant.

\subsection{Sharing self-monitoring information for prevention}

Each setting and context provides diverse possibilities for, and challenges to, performing self-care activities (Axelrod et al. 2009; Grönvall and Kyng 2012). For example, a person's emotional connection to a place and how he/she perceives himself/herself in that space may affect the willingness to self-monitor at a particular place. Hence, attitude is highly individual and may also depend on how people are currently populating the space in question. All the workshop participants had concerns about sharing illness-related information and performing measurement activities outside the house. For example, taking BP measurements at a friend's house or at a restaurant was considered unacceptable, even in a more private space, such as the restroom. It should be noted that the measurements were related to preventive monitoring, rather than monitoring of an identified illness. At the very least, this may have two effects: (1) the older workshop participants did not feel that whether or not they took their BP measurement, it was a matter of "life or death", and (2) workshop participants did not wish to address health-related issues (preventive care), if their activities (planned or not) might be misinterpreted as illness related. This was also confirmed by the qualitative study. For example, one participant stated "I'm not interested in coming off as ill, because I'm not".

In general, the respondents to the quantitative study were not willing to share their health information and preferred to not involve others in their health management. However, $69 \%$ of participants mentioned that they would like to share their data with their physicians, if needed. In contrast, workshop participants from the qualitative study shared the interpretation task with someone from their immediate family, for example a spouse, to verify whether a measurement had been correctly taken or not. 


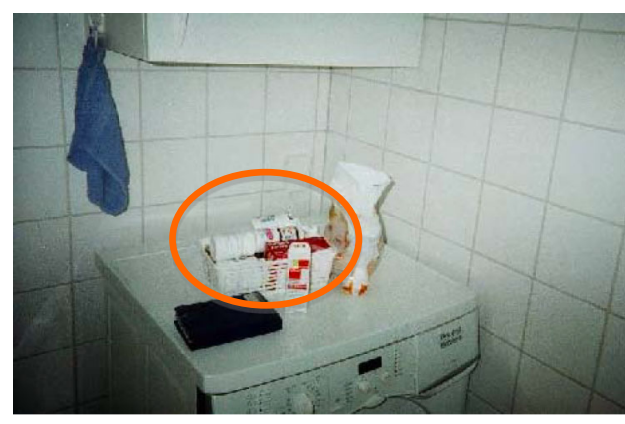

(a)

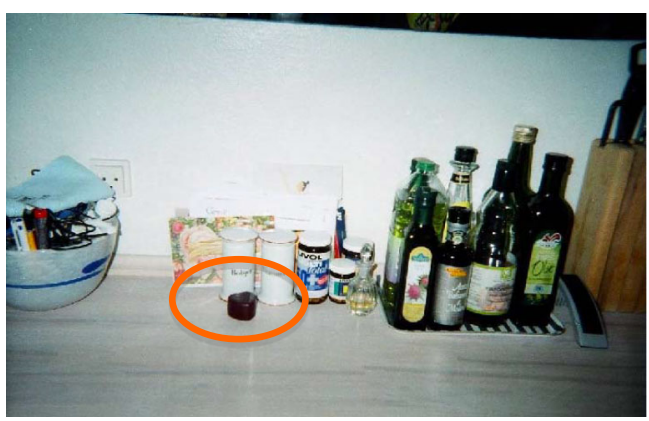

(b)

Fig. 6 Example of everyday objects distributed around the home: a the medication and $\mathbf{b}$ the heart-shaped box

\subsection{Motivational sources}

When the workshop participants understood the importance of BP measurements, they asked further questions and showed greater interest in our activities. In the project, we identified three main motivational sources. The first motivational source came from the individual and their desire for independence. For instance, a participant from the qualitative study stated, "To have to be dependent on others-I don't like that. I would rather be able to do it myself". In particular, the participants in the qualitative study expressed major concerns regarding the feeling of being independent. A participant said, "It is hard to have to depend on others, and to continue to do so".

The second motivational source derives from social relationships and a general interest in health. For instance, the qualitative study participants mentioned reasons for engaging in preventive self-monitoring. A female participant said that she and her companion would like to continue to be able to take care of their grandchildren, love each other, and live their lives. In the quantitative study, 42 participants had acquired a self-monitoring device because of a general interest in their health.

The third motivational source is related to the performance of daily activities. All participants from our study expressed a desire to continue to be able to do the things they normally do, and remain in their homes as long as possible. For instance, a participant from the qualitative study stated, "Going out and shovelling snow-I was surprised by how well it went. Our house is on a corner, so there was a lot of snow. But I wanted to do it! I am a little stubborn. I have to continue to be able to do the things I have always done". This was confirmed by the quantitative study, where 43 participants (of the 110 respondents that owned a self-monitoring device) used monitoring devices for prevention, as they would like to remain "as young as possible", and monitoring their health may help them to achieve that.

\subsection{The role of the doctor in prevention}

The quantitative study shows that $61 \%$ of the participants who use self-monitoring devices for prevention would like to replace existing healthcare services (e.g. counselling, doctor visits) with self-monitoring, whereas $76 \%$ of the participants who use self-monitoring devices for prevention would like to use them to supplement existing health services. Older adults are more reluctant to replace primary health care with selfmonitoring technology. However, the quantitative study shows that older adults are willing to acquire new self-monitoring technologies to maintain or improve their health ( $22 \%$ of the older respondents), but only after the technology has been on the market for a while and been tested (30\% of the older respondents). During preventive care activities, the role of the doctor may assume more of an advisory function. The study showed that workshop participants did not consider involving the doctor unless they were really ill, or their measured values were not within the normal range. Here, doctors can help in the interpretation of the measured values, or support and follow up on those who perform preventive care activities. Such activities might support older adults to get confident with preventive self-care activities. For instance, a participant from the qualitative study said, "The last two years we have been able to get our health checked through the company. But I always knew approximately how my health was, but it is always nice to be confirmed in your beliefs, and get a self-esteem boost. It also shows that the way I choose to live... that I don't really need to make any changes. By getting several check-ups, you can also see a trend, which tells me where I'm heading. That is a good indication".

\section{Design opportunities for preventive BP self- monitoring}

The findings indicate that current (BP) self-monitoring technology cannot fully support the needs of preventive self-monitoring in practice. For example, the complexity of 
the guidelines that must be followed to guarantee valid BP measurements, and the lack of contextual information may compromise the reliability of the measurements. Even though participants were able to take the (morning) measurements in a complex, distributed information space, such as the home, they failed to interpret and understand these measurements. Also, when designing self-monitoring Quantified Self tools or medical devices for bio-measurements, one has to understand the laws and regulations that are related to each form of measurement system and service. For example, there are more regulations related to medical devices, compared to consumer-segment Quantified Self services and tools. In this section, we discuss design opportunities for home-based self-monitoring that aim to enhance people's capacity for self-care, and promote preventive self-monitoring activities at home. Later, we present an example of how these design opportunities may enhance Quantified Self tools.

\subsection{Rethinking preventive self-monitoring of health parameters, in terms of a positive experience}

Most monitoring devices that have been designed to help people, including older adults, to monitor health parameters, to gain control of and/or prevent an illness or disability, originate in a clinician's perspective, rather than those performing self-monitoring (Morris et al. 2005; Storni 2010). As a consequence, to a great extent, current monitoring devices neglect the personal perceptions and motivation that make people control their health and wellbeing. Our study emphasizes that our participants have various motivational sources, which go beyond the actual self-measuring activity. In this sense, exploring how social aspects of people's everyday lives may increase the motivation to self-monitor [e.g. RunWithUs; Gil-Castiñeira et al. (2011)] and be a good strategy for supporting the selfmonitoring activity. The social environment is important, as many older adults want to be independent as long as possible, continue the activities they are used to, and to reside longer in their own homes. For example, our older project participants wanted to stay active and be able to visit friends, attend an activity centre, travel, or take care of their grandchildren. To not consider older adults' motivational factors might negatively influence their use of preventive self-monitoring in their everyday lives. Furthermore, sharing self-monitoring information plays a different role in prevention than in chronic disease management. Most of the workshop participants in our study considered preventive self-measurements as an individual effort that must be performed within the privacy of the home, and not in a social environment. The study participants feared they might be perceived as ill or weak, when they are not. Moreover, the complexity of the guidelines associated with bio-measurements turns the preventive self-measurement tasks into a complex endeavour that interferes with (existing) everyday activities that take place in and outside the home (a highly distributed information space with various operation centres). Hence, when considering the peculiar characteristics of preventive selfmonitoring, there is a need to provide older adults, and society in general, with a positive experience of self-measurement activities. Creating a positive experience may involve rethinking preventive self-monitoring as a tool that may support people in their achievement of personal goals, driven by their own motivation. A positive experience associated with the use of preventive self-monitoring technology would facilitate better integration of the monitoring activity and the older adults' daily routines. One way to promote such integration is to support the planning of the self-care activity, in this case, self-monitoring, as part of people's everyday lives (Dalgaard et al. 2013b). This integration would facilitate both older adults' participation in self-care activities and also that of younger adults who engage in tracking practices, and they should be considered from the outset of the design process (Grönvall and Kyng 2012). Indeed, as Bannon (1991) proposed more than 20 years ago, we should consider humans as actors, not factors. In society, considering self-monitoring a wellness activity rather than a disease-related activity may avoid stigmatization (Güldenpfennig and Fitzpatrick 2013) and help to create a positive experience that may increase the sharing of health information with close family members and friends.

\subsection{Supporting the active and informed individual}

In order to support the active and informed individual to monitor BP at home, healthcare technology designers should (re)consider the role of the individual using the technology (e.g. older adults) as proactive people (Rogers 2006). To support proactive people, a proper understanding of why they would follow a health intervention (e.g. using prescribed medication or self-monitoring) is necessary not only to enhance individual health awareness, but also to facilitate its integration into people's everyday lives (Dalgaard et al. 2013a). In our study, older adults seek to interpret, understand, and get meaning from the measurements related to their current health status. Thus, self-monitoring may support this learning activity (Dunlosky et al. 2007). In situations where self-monitoring promotes learning, the role of the doctor is shaped to support this interpretation process (preferred by some of our participants), or to confirm their personal interpretation and understanding of their current health status. To facilitate the interpretation and understanding of health data, visualization techniques may be used to provide older adults with the information needed to 
Fig. 7 a Line

chart visualization of BP; $\mathbf{b}$ data table visualization of $\mathrm{BP}$ readings

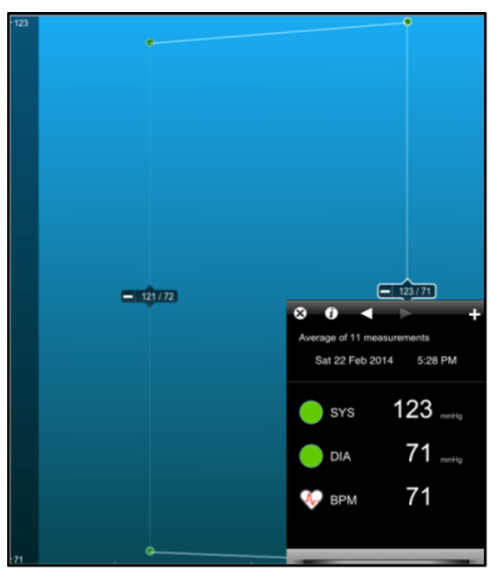

(a)

\begin{tabular}{|c|c|c|c|c|}
\hline & Date & sYs & DIA & BPM \\
\hline \multicolumn{5}{|c|}{ February 2014} \\
\hline 0 & Feb 22, 2014 5:40 PM & 128 & 84 & 70 \\
\hline e & Feb 22, 2014 5:39 PM & 150 & 83 & 70 \\
\hline$\theta$ & Feb 22, 2014 5:35 PM & 132 & 83 & 69 \\
\hline 0 & Feb 22, 2014 5:33 PM & 119 & 69 & 69 \\
\hline $\mathrm{O}$ & Feb 22, 2014 5:31 PM & 112 & 64 & 68 \\
\hline $\mathrm{O}$ & Feb 22, 2014 5:28 PM & 129 & 69 & 70 \\
\hline O & Feb 22, 2014 5:25 PM & 126 & 68 & 72 \\
\hline 0 & Feb 22, 2014 5:24 PM & 112 & 66 & 73 \\
\hline 0 & Feb 22, 2014 5:21 PM & 118 & 70 & 75 \\
\hline 0 & Feb 22, 2014 5:19 PM & 123 & 64 & 72 \\
\hline 0 & Feb 22, 2014 5:17 PM & 111 & 65 & 73 \\
\hline 0 & Average (Evening) & 123 & 71 & 71 \\
\hline
\end{tabular}

(b) improve health awareness (Lee and Dey 2011). For instance, our visualization activity reported that older adults rely on text values, rather than icons. They want to know the exact values, even if they do not understand their meaning. The workshop participants valued the visualizations, as they gave both an overview and details of BP data (see Fig. 3g). By presenting a combination of charts and a colour scheme representing BP levels (low, normal, borderline, high, and very high), visualizations would complement and enrich the actual BP values of an existing monitoring device (see Fig. 7a). A graphic interface that combines colourcoded and line charts (as suggested by participants-see Fig. 9) may provide a clear overview, embedding the temporal dimension (logging and making sense of BP data) to support individual self-reflection.

The lack of contextual information inherent to the monitoring devices provides opportunities for UbiComp and context-aware technologies to support self-monitoring of health data within and outside of a complex, distributed information space. The opportunities for UbiComp health technologies apply not only to chronic care (González et al. 2005), but also to preventive care, and outside a clinical setting. One way to address the lack of contextual information has been to proactively assess the level of movement and sound during the rest period, while taking the measurement, to inform about compliance with the measurement guidelines (Kusk et al. 2013). Furthermore, self-monitoring technology for preventive care may provide an opportunity for people to reflect on their health data, and help them to take action on their (current and future) health situation. For instance, existing efforts to establish guidelines for data collection that supports self-reflection (Li et al. 2011) on personal information are not yet reflected in, or available in, self-monitoring devices in older adults' homes. In contrast to proactive self-monitoring technology, we suggest supporting proactive individuals such as our proactive older adults, by providing a simple tagging functionality [e.g. TiY
(O'Murchu and Sigfridsson 2010)] for contextual information (e.g. whether the measurement was taken before or after breakfast or whether the person drank alcohol or coffee before the measurement), to document, reflect on, and understand the dynamic nature of the measurements. The tagging functionality could also serve as a tool to validate the reliability of the measured data and to enhance the users' awareness of their current health status. Thus, using visualization and tagging techniques may facilitate the interpretation of health data and support the active informed individual in learning about their health. All the aforementioned opportunities could support older adults' health education and self-learning activities related to preventive care.

\subsection{Implications of our findings for designing Quantified Self tools for BP Self-monitoring}

The findings and the above-mentioned design considerations may inform the design of medical devices, as well as the design of Quantified Self tools. Here, we discuss some implications of the foregoing aspects and how the design opportunities may be applied in the redesign of existing tools. On the one hand, Fig. 7 illustrates an example of a Quantified Self tool that enables the user to track BP readings. In this example, an overview of BP is provided through a simple chart (see Fig. 7a) and the details of the BP measurements through text (see Fig. 7b). On the other hand, typical medical BP measuring devices do not provide these functionalities, making it more difficult to understand the various measurements.

Even though the Quantified Self device informs the user of some of the relevant measurement guidelines to be taken before a measurement, they do not (similarly to medical devices) enforce the application of the measurement guidelines (Grönvall and Verdezoto 2013). For instance, in Fig. 8, we see an example of someone using a blood pressure measuring device while continuing his everyday 


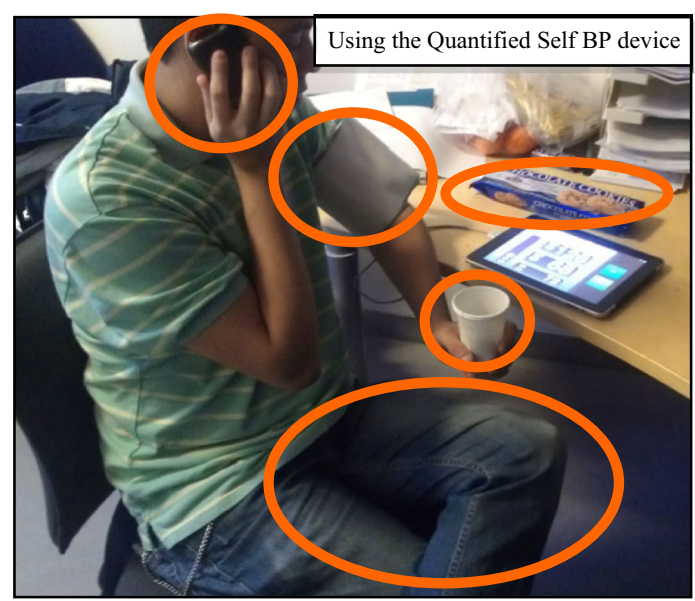

Fig. 8 Self-measurement with the Quantified Self BP measuring device in a non-clinical setting. Ellipses highlight elements that might hinder the reliability of BP measurements according to the measuring guidelines

activities. These everyday activities may hinder correct BP measurement. For example, to comply with the existing guidelines for recording a valid BP measurement, the individual should not be talking or crossing his legs during the measurement. Also, the person should rest for five minutes and avoid food, alcohol, and caffeine for $30 \mathrm{~min}$ prior to the measurement.

To support a positive experience of self-monitoring while still keeping the reliability of the measurement, the system should consider people's everyday activities and their individual motivations. In this sense, a calendar functionality (Dalgaard et al. 2013b) that integrates the self-monitoring activities with the user's other activities may help to promote a positive experience of taking a correct measurement. As a result, the self-monitoring activity may be planned, moved, and adjusted, according to people's everyday activities, supporting their personal motives.

To reflect on technology support for the active and informed individual, we introduce a number of mock-ups that emerged from our workshops and that materialize opportunities to consider some of the aforementioned relevant aspects, when designing non-clinical monitoring support. For example, to support individuals' self-learning and reflection, designers of health monitoring (preventive self-monitoring and Quantified Self) technology should address how best to enhance the visualization of overview data (see Fig. 7a) with various mechanisms (i.e. coded colour values) that may support the interpretation and understanding of the measured data. In this sense, for example, Fig. 9a illustrates the combination of a line chart and coded colours that may facilitate an understanding of the BP values, as explained above.
Furthermore, Fig. 9b also illustrates how the BP values are enhanced with various colours, to attach meaning to them. Regarding the tagging functionality, Fig. $9 \mathrm{~b}$ visualizes the activity of ingesting caffeine before measuring the blood pressure (see the blue oval in Fig. 9b), for example, to attach contextual information to measured values, and enhance self-reflection. In a Quantified Self context, rather than a purely preventive, self-monitoring situation, a tagging functionality might also support understanding of measured values, and document, for example, how various amounts of coffee may affect blood pressure in various situations.

Regarding the visualization of the details of BP data, Quantified Self tools usually present the information in two different ways. Figure 7a illustrates the average value when tapping on a specific measured value in the overview, by using text together with a specific colour, to add simple meaning to the value. Another representation of details is illustrated in Fig. 7b, in which all the measured values are listed, together with the average value. As suggested by our participants and explained above, the details of the selfmeasured values may be enhanced to add meaning, for example, by using colour-coded bars representing the various BP levels (see Fig. 10-low, normal, borderline, high, and very high). Additionally, the contextual information should also be presented in the vicinity of the measured values, to add necessary contextual information that supports learning and reflection (see the blue oval in Fig. 10).

\section{Conclusion}

In this paper, we have explored preventive self-monitoring activities by older adults in non-clinical settings. The study has focused primarily on BP measurements, but some of the findings presented herein may be applicable to other bio-monitoring scenarios. However, this should be further studied and understood, as people may use various selfmonitoring technologies and strategies for prevention.

Successful, home-based, preventive health monitoring may be highly beneficial for both the individual and society. However, our study reveals a number of aspects that may undermine the positive impact of individual health measurements. An important challenge is the integration of BP measurement guidelines into an individual's daily routines. With a successful integration, progress such as a better understanding of which life situations affect the body, both positively and negatively, may be achieved. To support the successful integration of health self-monitoring (e.g. blood pressure) into individuals' everyday lives, one has to consider personal motivational factors, the home as a distributed information space, and how health information 


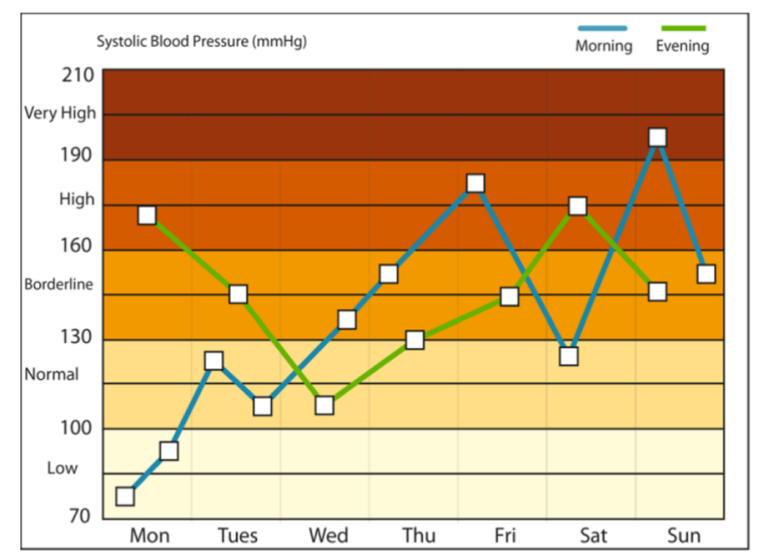

(a)

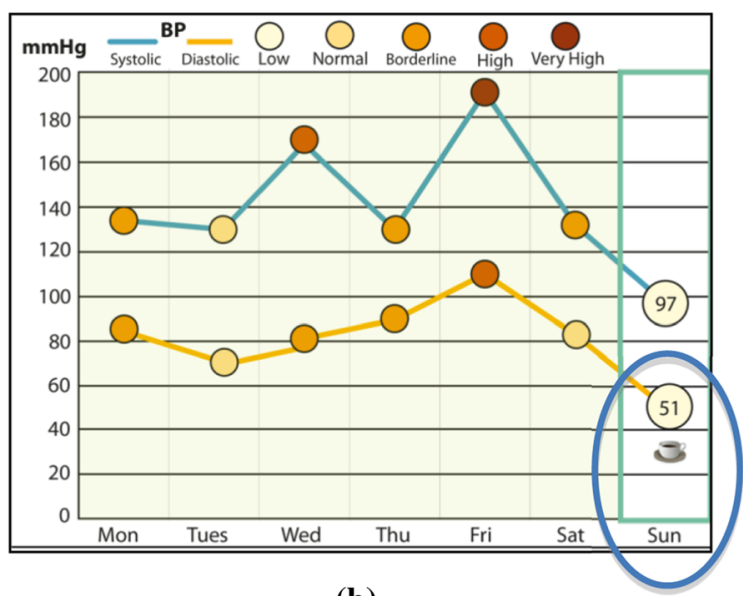

(b)

Fig. 9 a Line chart enhanced with a colour-coded scheme; b visualization of the tagging of contextual information

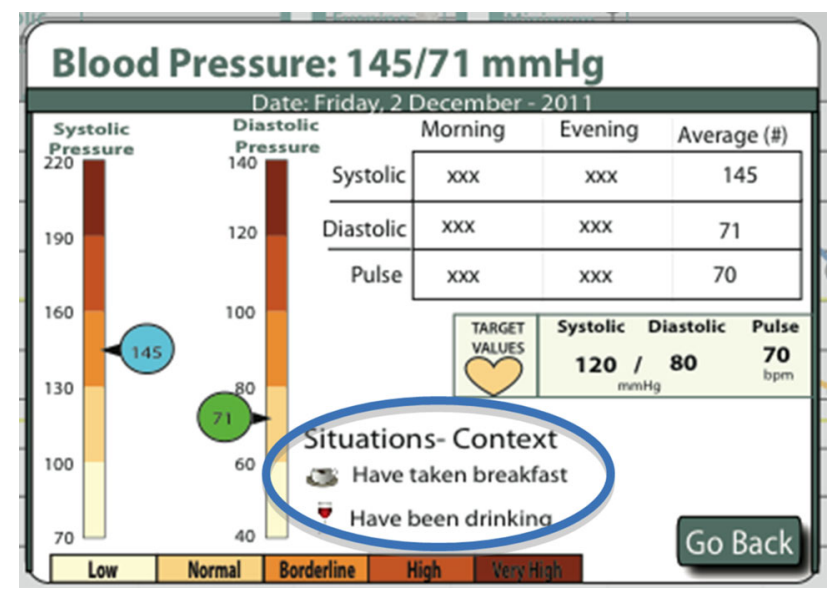

Fig. 10 Enhanced visualization of BP details with colour-coded bars and contextual information

is presented in non-clinical settings. For our study participants, a precise representation of the BP values was of utmost importance, to facilitate the interpretation and understanding of their health. Our findings call for rethinking preventive self-monitoring of BP (and possibly other health parameters), to generate a positive user experience by considering the individual's perspectives, needs, and desires, while keeping the reliability of the tracked information. One way to provide this positive experience is by supporting the active and informed individual, in order to improve older adults' health awareness, while supporting their independence at home. We also describe how our findings may inform the design of Quantified Self tools that have similar shortcomings to medical devices, for example lack of contextual information. The design opportunities presented in this paper may be transferrable to other age groups, as more people are becoming aware and starting preventive care activities before they get old.
Acknowledgments We would like to thank all participants in the Lev Vel Consortium, especially the older adults that took part in our project work. Lev Vel was funded by the Danish Council for Technology and Innovation and the Capital Region of Denmark. We would like to thank the reviewers for their comments, which strengthened our paper.

\section{References}

Ashiful A, Zarifi Eslami M, Sikkel K (2012) Elderly and homecare tasks: a literature review on problems Proc eHealth. IADIS Press, Italy

Axelrod L, Fitzpatrick G, Burridge J, Mawson S, Smith P, Rodden T, Ricketts I (2009) The reality of homes fit for heroes: design challenges for rehabilitation technology at home. J Assist Technol 3:35-43. doi:10.1108/17549450200900014

Baker RC, Kirschenbaum DS (1993) Self-monitoring may be necessary for successful weight control. Behav Ther 24:377-394

Ballegaard SA, Hansen TR, Kyng M (2008) Healthcare in everyday life: designing healthcare services for daily life. Paper presented at the proceedings of the SIGCHI conference on human factors in computing systems, Florence, Italy

Bannon L (1992) From human factors to human actors: the role of psychology and human-computer interaction studies in system design. In: Greenbaum J, Kyng $M$ (eds) Design at work. L. Erlbaum Associates Inc., Hillsdale, NJ, USA

Beaudin JS, Intille SS, Morris ME (2006) To track or not to track: user reactions to concepts in longitudinal health monitoring $\mathrm{J}$ Med Internet Res 8

Braun V, Clarke V (2006) Using thematic analysis in psychology Qualitative research in psychology 3:77-101

Choe EK, Lee NB, Lee B, Pratt W, Kientz JA (2014) Understanding quantified-selfers' practices in collecting and exploring personal data. Paper presented at the accepted in the SIGCHI conference on human factors in computing systems

Codagnone C (2009) Reconstructing the whole: present and future of Personal Health Systems Deliverable D 6

Coley C et al (1996) Preferences for home vs hospital care among low-risk patients with community-acquired pneumonia. Arch Intern Med 156:1565

Czeresnia D (1999) The concept of health and the difference between prevention and promotion. Cadernos de Saúde Pública 15:701-709 
Dalgaard LG, Grönvall E, Verdezoto N (2013a) Accounting for medication particularities: designing for everyday medication management. Paper presented at the international conference on pervasive computing technologies for healthcare, Venice, 5 May 2013

Dalgaard LG, Grönvall E, Verdezoto N MediFrame (2013) A tablet application to plan, inform, remind and sustain older adults' medication intake. In: Healthcare Informatics (ICHI), 2013 IEEE international conference on, 9-11 Sept 2013 pp 36-45. doi:10. 1109/ICHI.2013.12

Das A, Svanæs D (2010) Multiple perspectives on self-care: How can healthcare professionals and patients contribute to design? Therapeutic Strategies A Challenge for User Involvement in Design:9

Dunlosky J, Cavallini E, Roth H, McGuire CL, Vecchi T, Hertzog C (2007) Do self-monitoring interventions improve older adult learning? J Gerontol Ser B Psychol Sci Soc Sci 62:70-76

El Assaad MA, Topouchian JA, Asmar RG (2003) Evaluation of two devices for self-measurement of blood pressure according to the international protocol: the Omron M5-I and the Omron 705IT. Blood Press Monit 8:127-133

Epstein FH, Wei JY (1992) Age and the cardiovascular system. New Engl J Med 327:1735-1739

Fahmi PNA, Viet V, Deok-Jai C (2012) Semi-supervised fall detection algorithm using fall indicators in smartphone. Paper presented at the proceedings of the 6th international conference on ubiquitous information management and communication, Kuala Lumpur, Malaysia

Fitzpatrick G (2011) New challenges for health it—design fit for life. Paper presented at the Conference on design 4 health, Sheffield

Fox S, Duggan M (2013) Tracking for health. Pew Research Center's Internet and American Life Project

Gil-Castiñeira F, Fernández-López A, Bravo CL, Cid-Vieytes N, Conde-Lagoa D, Costa-Montenegro E, González-Casta FJ (2011) RunWithUs: a social sports application in the ubiquitous Oulu environment. Paper presented at the proceedings of the 10th international conference on mobile and ubiquitous multimedia, Beijing, China

Glasgow RE, Tracy Orleans C, Wagner EH, Curry SJ, Solberg LI (2001) Does the chronic care model serve also as a template for improving prevention? Milbank Q 79:579-612

González VM, Tentori ME, Morán EB, Favela J, Martínez AI (2005) Understanding mobile work in a distributed information space: implications for the design of ubicomp technology. In: Proceedings of the 2005 Latin American conference on Humancomputer interaction, 2005. ACM, pp 52-63

Grönvall E, Kyng M (2012) On participatory design of home-based healthcare Cognition, Technology and Work:1-13 doi:10.1007/ s10111-012-0226-7

Grönvall E, Verdezoto N (2013) Beyond self-monitoring: understanding non-functional aspects of home-based healthcare technology. Paper presented at the 2013 ACM international joint conference on pervasive and ubiquitous computing (UbiComp 2013), Zurich, Switzerland

Güldenpfennig F, Fitzpatrick G (2013) A monitoring device as assistive lifestyle technology: combining functional needs with pleasure. In: Proceedings of the 4th augmented human international conference, 2013. ACM, pp 190-193

Hood L, Flores M (2012) A personal view on systems medicine and the emergence of proactive $\mathrm{P} 4$ medicine: predictive, preventive, personalized and participatory. New Biotechnol 29:613-624. doi:10.1016/j.nbt.2012.03.004

Juel K, Sørensen J, Brønnum-Hansen H (2008) Supplement: risk factors and public health in Denmark. Scand J Public Health 36:1-227. doi:10.1177/1403494800801101
Kahneman D, Krueger AB, Schkade DA, Schwarz N, Stone AA (2004) A survey method for characterizing daily life experience: the day reconstruction method. Science 306:1776-1780. doi:10. 1126/science. 1103572

Kane RL, Kane RA, Arnold SB (1985) Prevention and the elderly: risk factors. Health Serv Res 19:945

Klasnja P, Consolvo S, Pratt W (2011) How to evaluate technologies for health behavior change in HCI research. Paper presented at the proceedings of the 2011 annual conference on human factors in computing systems, Vancouver, BC, Canada

Kusk K, Nielsen DB, Thylstrup T, Rasmussen NH, Jorvang J, Pedersen CF, Wagner S (2013) Feasibility of using a lightweight context-aware system for facilitating reliable home blood pressure self-measurements. In: Pervasive computing technologies for healthcare (PervasiveHealth), 2013 7th international conference on, 5-8 May 2013. pp 236-239

Lee ML, Dey AK (2010) Embedded assessment of aging adults: A concept validation with stakeholders. In, 2010. IEEE, pp 1-8

Lee ML, Dey AK (2011) Reflecting on pills and phone use: supporting awareness of functional abilities for older adults. Paper presented at the Proceedings of the 2011 annual conference on Human factors in computing systems, Vancouver, BC, Canada

Li I, Dey A, Forlizzi J (2010) A stage-based model of personal informatics systems. Paper presented at the Proceedings of the SIGCHI Conference on Human Factors in Computing Systems, Atlanta, Georgia, USA

Li I, Dey AK, Forlizzi J (2011) Understanding my data, myself: supporting self-reflection with ubicomp technologies. Paper presented at the Proceedings of the 13th international conference on Ubiquitous computing, Beijing, China

Lupton D (2012) M-health and health promotion: the digital cyborg and surveillance society Soc Theory. Health 10:229-244

Lupton D (2013a) The digitally engaged patient: self-monitoring and self-care in the digital health era Soc Theory. Health 11:256-270. doi:10.1057/sth.2013.10

Lupton D (2013b) Quantifying the body: monitoring and measuring health in the age of mHealth technologies. Crit Public Health 23:393-403. doi:10.1080/09581596.2013.794931

Lupton D (2013c) Understanding the human machine [commentary]. Technol Soc Mag IEEE 32:25-30. doi:10.1109/mts.2013. 2286431

Mallery L, Rockwood K (1992) Preventive Care for the Elderly: uncovering the unmet needs of this population. Can Fam Physician 38:2371

Mik-Meyer N, Villadsen K (2007) Magtens former: Sociologiske blikke på statens møde med borgeren. Sociologiske perspektiver på statens møde med borgeren, Hans Reitzels Forlag

Montgomery-Downs H, Insana S, Bond J (2012) Movement toward a novel activity monitoring device. Sleep Breath 16:913-917. doi:10.1007/s11325-011-0585-y

Morris M, Intille S, Beaudin J (2005) Embedded assessment: Overcoming barriers to early detection with pervasive computing Pervasive computing:333-346

O'Brien E et al (2003) European Society of Hypertension recommendations for conventional, ambulatory and home blood pressure measurement. J Hypertens 21:821

O’Murchu N, Sigfridsson A (2010) TiY (tag-it-yourself). Paper presented at the Proceedings of the 8th ACM Conference on Designing Interactive Systems, Aarhus, Denmark

Parati G et al (2010) European society of hypertension practice guidelines for home blood pressure monitoring. J Hum Hypertens 24:779-785

Rivera-Pelayo V, Zacharias V, Müller L, Braun S (2012) Applying quantified self approaches to support reflective learning. In: 
Proceedings of the 2nd International Conference on Learning Analytics and Knowledge, 2012. ACM, pp 111-114

Rogers Y (2006) Moving on from Weiser's vision of calm computing: engaging UbiComp experiences. In: Dourish P, Friday A (eds) UbiComp 2006: Ubiquitous Computing, vol 4206. Lecture Notes in Computer Science. Springer Berlin Heidelberg, pp 404-421. doi:10.1007/11853565_24

Smarr L (2012) Quantifying your body: a how-to guide from a systems biology perspective. Biotechnol J 7:980-991

Sørensen TI, Pedersen BK, Sandbæk A, Overvad K (2013) Skal overvægtige voksne tabe sig? Vidensråd For Forebyggelse. Available from http://www.vidensraad.dk/sites/default/files/ vidensraad_overvaegt_digital_02.pdf. Accessed 29 Oct 2013

Storni C (2010) Multiple Forms of Appropriation in Self-Monitoring Technology: reflections on the Role of Evaluation in Future SelfCare. Int J Human-Comput Int 26:537-561. doi:10.1080/ 10447311003720001

Storni C (2013) Design challenges for ubiquitous and personal computing in chronic disease care and patient empowerment: a case study rethinking diabetes self-monitoring Personal and Ubiquitous Computing:1-14 doi:10.1007/s00779-013-0707-6

Stults BM (1984) Preventive health care for the elderly. West J Med 141:832

Swan M (2012) Sensor mania! the internet of things, wearable computing, objective metrics, and the Quantified Self 2.0. J Sens Actuator Networks 1:217-253

Swan M (2013) The Quantified Self: Fundamental Disruption in Big Data Science and Biological Discovery. Big Data 1:85-99
Tentori M, Hayes GR, Reddy M (2012) Pervasive computing for hospital Chronic, and Preventive Care found trends. HumComput Interact 5:1-95. doi:10.1561/1100000024

Turner-McGrievy GM, Beets MW, Moore JB, Kaczynski AT, BarrAnderson DJ, Tate DF (2013) Comparison of traditional versus mobile app self-monitoring of physical activity and dietary intake among overweight adults participating in an mHealth weight loss program. J Am Med Inform Assoc 20:513-518. doi:10.1136/amiajnl-2012-001510

Verdezoto N, Grönvall E, Vincentz S, Rostgaard Thielsen S (2012) Exploring challenges of self-monitoring for senior adults. Workshops on bridging clinical and non-clinical health practices: opportunities and challenges at CHI 2012, May 5-10, Austin, Texas

Verdezoto N, Nunes F, Grönvall E, Fitzpatrick G, Storni C, Kyng M (2014) Designing self-care for everyday life. Paper presented at the extended abstract at the 8th Nordic Conference on HumanComputer Interaction

Wagner EH (1998) Chronic disease management: what will it take to improve care for chronic illness? Effective Clin Pract ECP 1:2-4

WHO (2003) Adherence to long-term therapies Evidence for action. World Health Organization, Geneva

Wiederhold BK (2012) Self-Tracking: better medicine through pattern recognition cyberpsychology. Behav Soc Netw $15: 235-236$

Yau N, Schneider J (2009) Self-Surveillance. Bull Am Soc Inf Sci Technol 35:24-30. doi:10.1002/bult.2009.1720350507 